\title{
The Role of External Cues in Korean Mirror Writing
}

\author{
HyangHee Kim $^{\mathrm{a}}$ Kyungmi Oh $^{\mathrm{b}}$ Chin-Sang Chung ${ }^{\mathrm{c}}$ \\ ${ }^{a}$ Department of Rehabilitation Medicine and Graduate Program in Speech and Language Pathology, Yonsei \\ University College of Medicine, ${ }^{b}$ Department of Neurology, Korea University Anam Hospital, and ' Department of \\ Neurology, Samsung Medical Center, Sungkyunkwan University School of Medicine, Seoul, South Korea
}

Dear Sir,

In this report, we describe a case of pathological mirror writing involving a right-handed Korean patient with a left basal ganglia lesion. Using his intact left hand, he always initiated writing from the right margin, and each grapheme was written as its mirror image with intact writing sequences. However, with external verbal ('Please write rightwards from here') and visual (rightward arrow) cues, he wrote normally from the left edge of the paper. This finding confirms that people with basal ganglia lesions compensate for a malfunction by relying on external cues to guide movements. A particularly interesting observation is that, with only a verbal cue to write rightwards, writing initiated from the left margin produced a vertical script with mirror writing. This phenomenon is noteworthy because it can be observed only in languages with a topto-bottom writing system.

\section{Case Report}

A 63-year-old, right-handed man with a college education was admitted with a cerebral infarction. Brain MRI showed an acute infarction at the left basal ganglia and a subclinical lesion at the right thalamus (fig. 1). He had not manifested any clinical signs or symptoms pertaining to the thalamic lesion before admission. Neurological findings at the time of admission included right central facial palsy, mild dysarthria, and right hemiplegia. At the

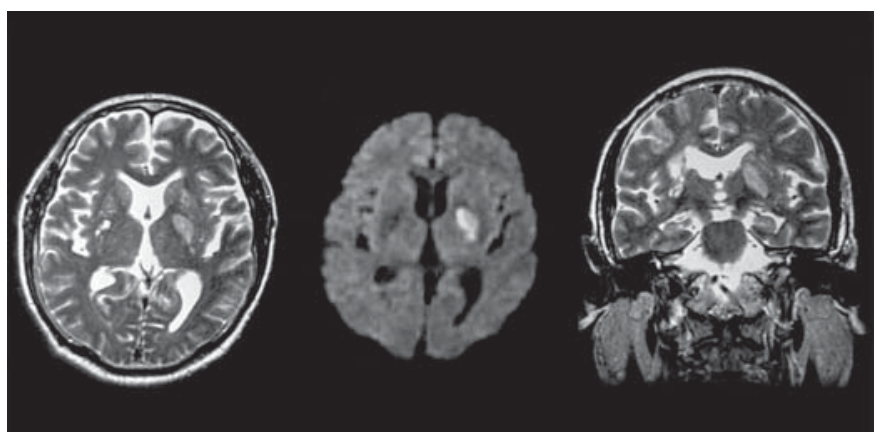

Fig. 1. Magnetic resonance imaging of the brain. The images from the left are: $\mathrm{T}_{2}$-weighted axial image, a diffusion axial image and a $\mathrm{T}_{2}$-weighted coronal image, showing an acute infarction at the left basal ganglia and a subclinical lesion at the right thalamus.

time of language evaluation on the 8th day after onset, the patient manifested relatively fluent utterances, and intact auditory comprehension, repetition, and naming abilities. He was able to read simple, short sentences without mirror reading. However, mirror writing with his intact left hand was observed for individual Korean graphemes when he wrote his name and address spontaneously, and also when he wrote words, phrases, and sentences dictated to him. He was able to acknowledge the difference between mirror and normal writing on twenty pairs of written stimulus cards without any hesitation. He did not have difficulty in drawing figures such as a rectangle or a human face. Calculations were performed accurately and he could identify his own right and left body parts. We could not test his righthanded writing because he presented with hemiplegia. He has never been left-handed and none of his family members was known to be left-handed.

We asked the patient to write dictated text, to copy normally written stimuli, and to copy mirror image stimuli. In all task situations, he mirror wrote all graphemes and the script direction was reversed such that he demonstrated right-to-left writing. Without prompting, he wrote the sentence '나는 밥을 먹는다' (meaning 'I eat a meal')

\section{KARGER}

Fax +41613061234 E-Mail karger@karger.ch www.karger.com
(C) 2007 S. Karger AG, Basel 0014-3022/08/0592-0085\$24.50/0

Accessible online at: www.karger.com/ene
HyangHee Kim, PhD, Associate Professor

Department of Rehabilitation Medicine

Yonsei University College of Medicine, Shinchon-dong 134, Seodaemun-gu

Seoul 120-752 (Republic of Korea)

Tel. +82 22228 3900, Fax +82 22227 7578, E-Mail h.kim@yonsei.ac.kr 
a

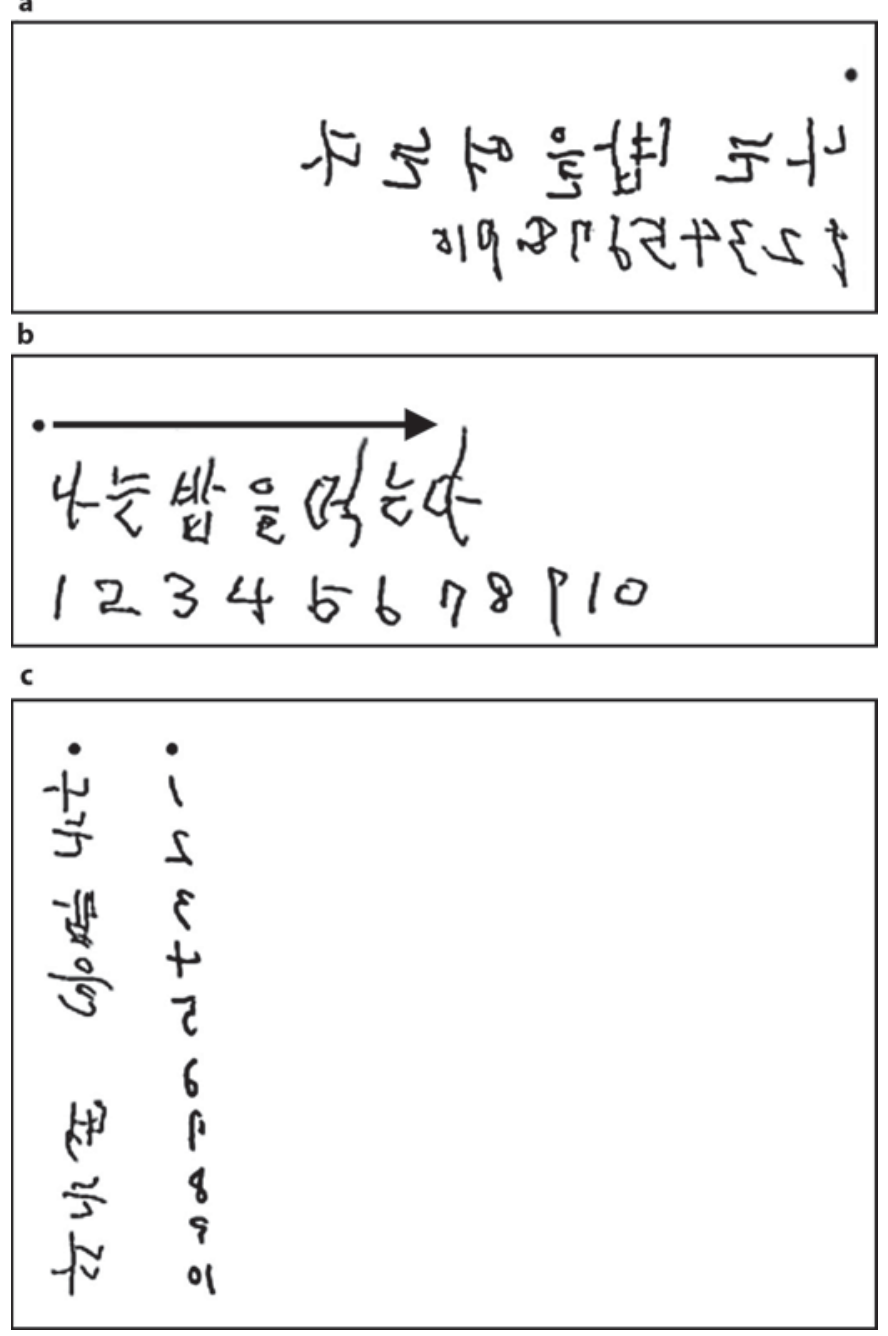

Fig. 2. Examples of a sentence and the Arabic numbers (1-10) written by the patient after different external cues. a Without prompting, the patient displayed mirror-writing starting from the right edge of the paper. $\mathbf{b}$ With both a verbal and a visual cue to write from the left to the right, the patient wrote correctly without mirror writing. c With only a verbal cue to start writing on d

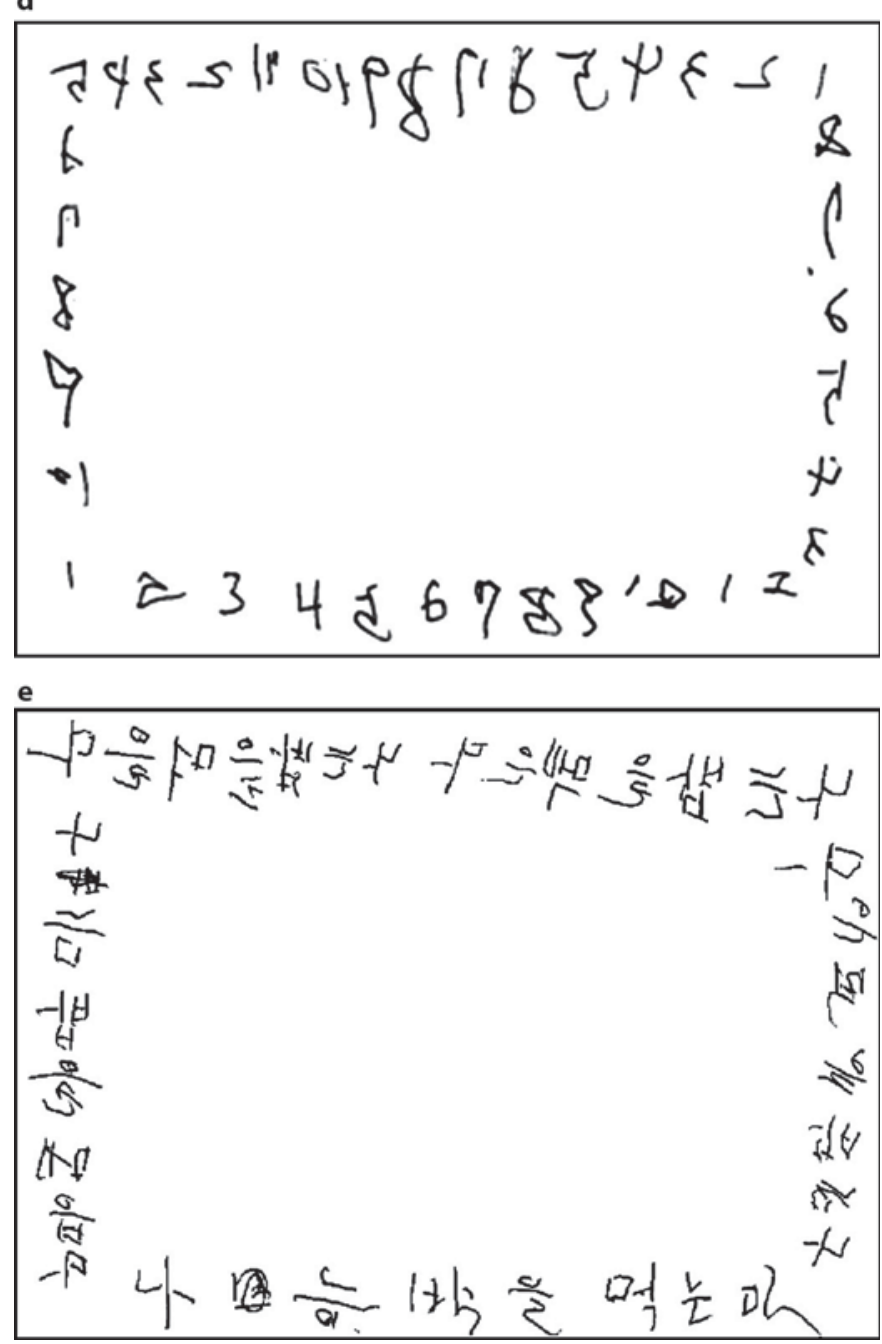

the left edge of the paper, the patient wrote the sentence downwards in mirror writing. d, e With the verbal instruction, 'Please start writing numbers from 1' or 'Please write a sentence', the Arabic numbers (1-10) and the sentence '나는 밥을 먹는다' were written around the edge of the paper starting at the right upper corner. $\rightarrow=$ Direction of writing; $\bullet$ = reference point. and the Arabic numbers (1-10) in mirror writing (fig. 2a). However, as shown in figure $2 \mathrm{~b}$, he was able to correct the mirror writing when external verbal and visual cues were given, and did so without any difficulty or hesitation. The cues given to the patient were firstly verbalized: 'Please write starting at this point and you must write rightwards' as the researchers pointed to the left edge of the paper as reference, and secondly providing the visual cue of a rightward arrow as the researchers specified the writing direction. When we did not provide the 'rightward arrow' visual cue, the patient wrote downward along the left edge of the paper with mirror writing of each grapheme (fig. 2c). To further demonstrate this phenomenon, we asked the patient to write Arabic numbers (1-10) and a sentence along the edge of the paper, starting at the right upper corner with the verbal instruction, 'Please start writing numbers from 1' or 'Please write the sentence '나는 밥을 먹는다' (meaning 'I eat a meal') (fig. $2 \mathrm{~d}, \mathrm{e}$ ). In this case, the patient initiated and continued mirror writing along the edges until he started to write normally at the left lower corner. Mirror writing resumed when he wrote from bottom to top along the right hand side.

\section{Discussion}

With a left basal ganglia lesion, our patient initiated writing at the right margin of the paper. Upon initiating writing, the patient had to write proper, nonmirror writing with the inevitable tendency of abiding by 'motor sequences' dictated by learned 'motor programming'. That is 
to say, no matter where one initiates writing on the paper, he or she will abide by the intact motor programming sustaining graphemic and syllabic writing 'sequences' $[1,2]$. This finding confirms the assertion by Schott [3] that mirror writing is a phenomenon associated with movement.

The Korean patient could modify the mirror writing when provided with external cues. The use of both auditory and visual external cues was effective in our patient, demonstrating that the presentation of external cues can correct impaired, automatic control in patients with a basal ganglia lesion. The influence of external cues on behavior has previously been reported in basal ganglia dysfunction [4]. Interestingly, without the visual cue, the directional modification of writing to the right was not induced. In this situation, the strong tendency to write to the left was not suppressed and, as there was no space towards the left on the paper, he wrote downwards along the left edge of the paper. Similarly, it has been reported that auditory cues have a lesser effect than visual cues on limb movement, such as gait initiation in people with Parkinson's disease [5].

The downwards writing observed in this patient implies that characteristics of mirror writing at the level of a connected script are language-specific $[2,6,7]$. The modern Korean script is typically written in the left-to-right direction, as are IndoEuropean scripts such as English. Howev- er, columnar, top-to-bottom writing was the primary Korean writing script style until some decades ago, and is still permitted today. Korean top-to-bottom writing is feasible because the Korean syllabic writing system consists of spatially well-formed syllabic blocks (e.g. '한글' / han-geul/, meaning 'Korean alphabet'). Each block (i.e. '한' /han/, '글'/geul/) contains one to three consonants (' $\overline{0}$ ' $/ \mathrm{h} /$, ' $\llcorner$ ' $/ \mathrm{n} /$, in the first syllabic block of the example) and one vowel (' $\vdash$ ' $/ \mathrm{a} /$ ) out of the 24 letters (14 consonants and 10 vowels) in the Korean alphabet.

This unique phenomenon of mirror writing at the script level was accentuated when the patient was asked to write Arabic numbers and a sentence along the edges of the paper. When he wrote leftwards from the right top margin and downwards at the left margin, he showed mirror writing. However, normal writing appeared at the bottom edge of the paper while writing rightwards, and mirror writing was resumed when he wrote bottom-to-top. In a follow-up contact with the patient, he reported that his left-handed writing no longer displayed mirror writing.

\section{Acknowledgement}

The authors are grateful to Dr. Geoffrey Schott, UCL Hospitals, National Hospital for Neurology and Neurosurgery, Queen Square, London, UK, for his helpful comments and suggestions on the earlier draft of the manuscript.

\section{References}

1 Chan J, Ross E: Left-handed mirror writing following right anterior cerebral artery infarction: evidence for non-mirror transformation of motor programs by right supplementary motor area. Neurology 1988;38: 59-63.

2 Chia L, Kinsbourne M: Mirror-writing and reversed repetition of digits in a right-handed patient with left basal ganglia haematoma. J Neurol Neurosurg Psychiatry 1987;50: 786-788.

3 Schott GD: Mirror movements of the left arm following peripheral damage to the preferred right arm. J Neurol Neurosurg Psychiatry $1980 ; 43: 768-773$.

4 Cunnington $\mathrm{R}$, Iansek $\mathrm{R}$, Bradshaw JL: Movement-related potentials in Parkinson's disease: external cues and attentional strategies. Mov Disord 1999;14:63-68.

5 Jiang Y, Norman KE: Effects of visual and auditory cues on gait initiation in people with Parkinson's disease. Clin Rehabil 2006; 20:36-45.

6 Schott GD, Schott JM: Mirror writing, lefthandedness and leftward scripts. Arch Neurol 2004;61:1849-1851.

7 Tashiro K, Matsumoto A, Hamada T, Moriwaka F: The aetiology of mirror writing: a new hypothesis. J Neurol Neurosurg Psychiatry 1987;50:1572-1578. 